\title{
BIO-Young: Smartphone-based biomarkers in adolescents with unipolar disorder and bipolar disorder, their healthy siblings and healthy controls
}

\author{
Sigurd Melbye*, Maria Faurholt-Jepsen*, Mads Frost**, Jakob Bardram***, Lars Vedel Kessing* \\ "Psychiatric Center Copenhagen, Copenhagen University Hospital, Rigshospitalet, Blegdamsvej 9, Copenhagen, Denmark \\ ${ }^{* *}$ Monsenso, Denmark \\ *** Department of Applied Mathematics and Computer Science, and Copenhagen Center for Health Technology, Technical University of Denmark, Lyngby, Denmark
}

\section{BACKGROUND}

MENTAL DISORDERS

Mental disorders are prevalent in youth. Among patients suffering from mental disorders $50 \%$ develop symptoms before the age of 14 years, and $75 \%$ before the age of 25.

Approximately $20 \%$ of patients with unipolar and bipolar disorder have onset of symptoms before the age of 25 [1].

A majority of affective disorders are not recognized, diagnosed, nor treated adequately [2]. Delay of treatment reduces the chances of obtaining remission substantially [3].

It is of crucial importance to be able to discriminate unipolar depressive disorder from bipolar disorder in clinical practice, as treatment course of illness and outcome differ between the two disorders [4]

It would be of great interest to add objective measures that can assist in the discrimination between the two disorders.

\section{SMARTPHONES}

Smartphones provide a unique platform for continuous real-time and fine-grained monitoring. Equipped with powerful sensing, computation and communication capabilities, smartphones can continuously monitor an individual's physical activity, location and environment.

Depression and mania are associated with several behavioral components and motivational states, some of which may be detectable using smartphone sensors.

\section{PRIOR RESEARCH}

Prior research by our research group done in the MONARCA project have developed and tested an unique smartphone-based system for monitoring and treatment of adult patients with bipolar disorder. The system allows for collection of self-assessment data and objective sensor data from patients on a daily basis.

The system has been found highly usable and useful by patients with a high self-assessment adherence $(87-95 \%)$, helped patients to better manage their disease, and automatically generated objective smartphone data correlated with the severity of depression and mania [5].

Although onset of unipolar and bipolar disorder frequently occurs in adolescence, and the use of smartphones is appealing in youth, no study has investigated the use of smartphones in the diagnosis and monitoring of illness in adolescence patients with affective disorder.

\section{OBJECTIVES}

To identify a composite smartphone-based electronic marker that

1: Discriminates adolescent patients with unipolar disorder from adolescent patients with bipolar disorder during depression and remission.

2: Discriminates adolescent patients with unipolar and adolescent patients with bipolar disorder from their adolescent healthy first generation siblings and adolescent healthy control individuals without a family history of affective disorder.

3: Discriminates between manic states, depressive states and euthymic states in adolescent patients with bipolar disorder and between depressive and euthymic states in adolescent patient with unipolar disorder.
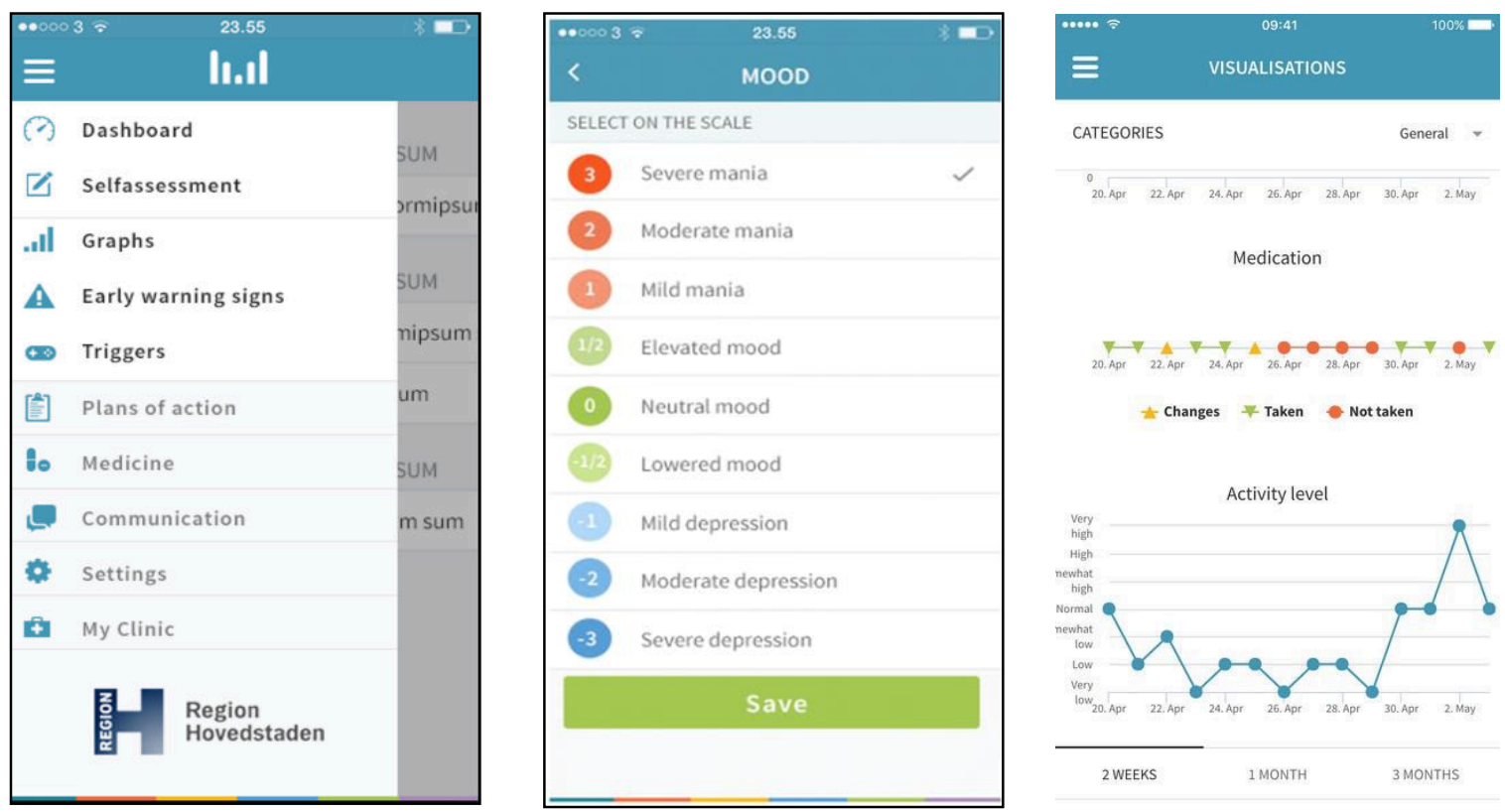

Figures: Screenshots from the Monsenso smartphone app. Participants self-
In addition, the smartphones collect automatically generated objective data

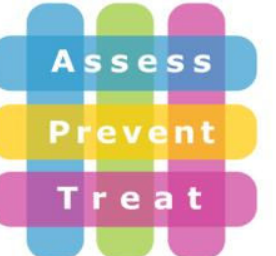

- The study includes the following individuals between 15 and 25 years of age:

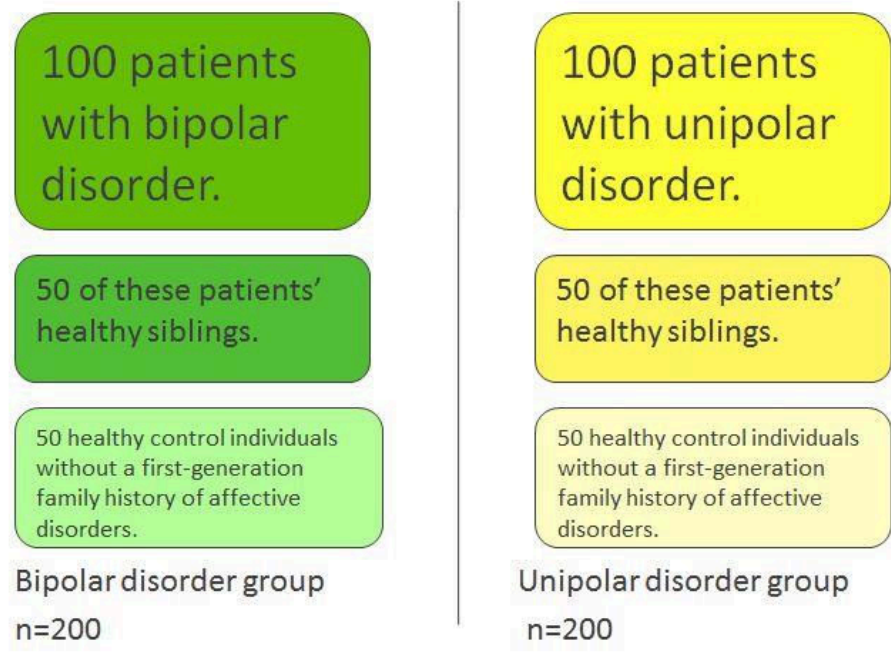

METHODS

DIAGNOSIS

Diagnostic assessment will be done using the Schedules for Clinical Assessment in Neuropsychiatry interview (SCAN) categorizing patients into unipolar disorder and bipolar disorder type I or II.

CUNICAL ASSESSMENT

Clinical assessment will be done using the Hamilton Depression Scale-17, the Young Mania Rating Scale and the Functional Assessment Short Test.

SMARTPHONE

All participants in the study will use the Monsenso smartphone app for at least 3 months.

\begin{tabular}{|l|l|}
\hline \multicolumn{2}{|c|}{ Smartphone-based objective data } \\
\hline Social activity & $\begin{array}{l}\text { In- and outgoing phone calls and text } \\
\text { messages }\end{array}$ \\
\hline $\begin{array}{l}\text { Physical } \\
\text { activity } \\
\text { Mobility }\end{array}$ & Step counter \\
\hline Speech & Location estimation \\
\hline
\end{tabular}

INNOVATION AND ORIGINALITY

This is the first study to

1. Investigate the use of a composite smartphone-based electronic marker in adolescent patients with unipolar disorder and adolescent patients with bipolar disorder.

2: Measure smartphone-based electronic markers in adolescents with unipolar disorder and bipolar disorder and their healthy siblings.

3: Investigate whether smartphone-based electronic markers discriminates between adolescent patients with unipolar disorder and adolescent patients with bipolar disorder and between patients with affective disorders and healthy relatives at risk and healthy control individuals.

\section{RESULTS/STATUS}

As of today the project has not reached through the stage of recruitment and data collection. It is currently too early to interpret the data or make suggestive hypotheses.

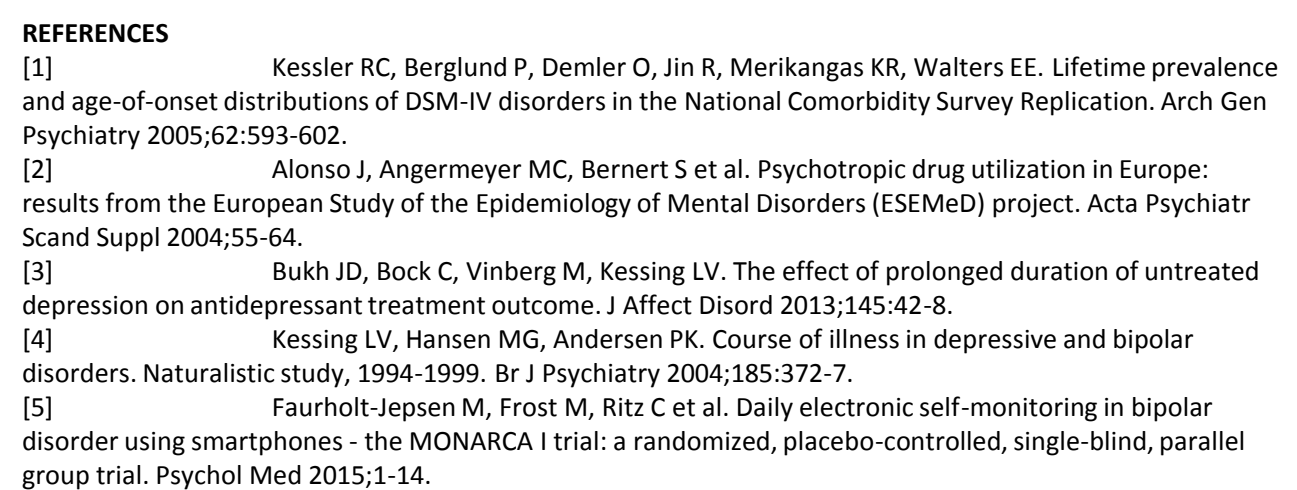

